\title{
Skeletal Radiology: the year in review 2019
}

\author{
Mark J. Kransdorf ${ }^{1} \cdot$ Thomas M. Link $^{2}$ - William E. Palmer ${ }^{3}$. Valerie A. Fitzhugh ${ }^{4}$
}

Received: 14 April 2020 / Revised: 7 May 2020 / Accepted: 10 May 2020 / Published online: 28 May 2020

(C) ISS 2020

\begin{abstract}
In 2019, Skeletal Radiology published a total of 253 articles including 111 scientific articles, 36 review articles, 51 case reports, 16 Test-Yourself cases, 15 technical reports, as well as multiple browser notes, meeting abstracts, and meeting summaries. As we have done previously, in this review, we will highlight those articles that stimulated the most interest from our readers, as measured by their downloads, and those that stimulated other researchers and authors, as measured by their citations. The manuscripts highlighted herein were chosen from this list.
\end{abstract}

The year 2019 was a banner year for the Journal, with a record of 1032 manuscript submissions: an increase of more than 5\% over the previous year. In years past, the number of manuscripts that could be published was determined by the number of pages allowed per issue by the publisher. With the change to primarily digital media, we are now limited by the number of manuscripts per issue; currently set at 21 manuscripts for each issue of Skeletal Radiology, yielding a yearly total of 252 manuscripts. While that sounds quite rigid, there is some flexibility and last year, for example, we published 253 articles, for an overall acceptance rate just below $25 \%$. Not surprisingly, scientific articles were most numerous, representing $44 \%$ of published manuscripts, followed by case reports $(20 \%)$, review articles (14\%), test-yourself cases, and technical reports $(6 \%$ each). The final $10 \%$ of submissions included browser notes, meeting abstracts and highlights, perspectives, and last but not least, the 2018 Year in Review!

The range of the published subject matter in 2019 was quite remarkable: from basic radiologic-pathologic correlation to artificial intelligence. As we have done previously, in this

Mark J. Kransdorf

kransdorf.mark@mayo.edu

1 Department of Radiology, Mayo Clinic, 5777 E Mayo Boulevard, Phoenix, AZ, USA

2 Department of Radiology and Biomedical Imaging, University of California San Francisco, San Francisco, CA, USA

3 Department of Radiology, Massachusetts General Hospital, Boston, MA, USA

4 Department of Pathology and Laboratory Medicine, Rutgers, The State University of New Jersey, New Brunswick, NJ, USA review, we will highlight those articles that stimulated the most interest from our readers, as measured by their downloads, and those that stimulated other researchers and authors, as measured by their citations. While one may assume such selection criteria would be biased by the publication date, favoring those published early in the year; however, that is generally not the case, due at least in part to the fact that most articles are published online several months prior to their print appearance. The most downloaded article for the year was published in the October issue and online 6 months earlier. This paper was downloaded a remarkable 11,962 times by the end of the year and 12, 919 times by the end of January of this year. This review article by Balius et al. [1], titled "Sonographic landmarks in hamstring muscles," is a systematic approach to the ultrasound evaluation of the hamstring muscles, dividing them into four areas of interest based on constant, reproducible, and characteristic landmarks. With exquisitely labeled clinical and sonographic images, this systematic approach to evaluation will serve both the experienced and novice musculoskeletal sonographer.

The next most downloaded manuscript was also a review article. This manuscript, by Robert Kwee and Thomas Kwee [2], titled "Calcified or ossified benign soft tissue lesions that may simulate malignancy," reviewed the spectrum of these mineralized benign soft tissue lesions. The authors emphasize and illustrate the importance of distinguishing calcification from ossification, as well as the significance of the clinical presentation and specific imaging features when making a diagnosis or developing an appropriately limited differential. Another highly downloaded review was by Fields and colleagues [3], titled "Adhesive capsulitis: review of imaging findings, pathophysiology, clinical presentation, and treatment 
options." As the authors note in their comprehensive review, while adhesive capsulitis has historically been considered a clinical diagnosis, we are now able to identify MR and ultrasound imaging features that will aid in the appropriate diagnosis and treatment of this condition: features that are wellexplained and illustrated in this review.

Another highly downloaded scientific article was titled "Artificial intelligence-assisted interpretation of bone age radiographs improves accuracy and decreases variability," by Tajmir et al. [4]. In this study, researchers compared the bone age assessment performance on a cohort of 280 age and gender-matched bone age radiographs by pediatric radiologists, both without and then with, artificial intelligence assistance. The authors used a previously developed automated artificial intelligence deep-learning algorithm to perform bone age assessment using convolutional neural networks. The study showed that artificial intelligence improved the radiologist's bone age assessment by increasing accuracy and decreasing variability and error. In the final analysis, the use of artificial intelligence by radiologists improved performance compared to artificial intelligence alone, a radiologist alone, or a pooled cohort of experts. In addition to being one of the most highly downloaded studies, this was also the most cited study, receiving 7 citations in 2019, and 11 as of this writing.

The second most cited manuscript was also a scientific article addressing the use of artificial intelligence, titled "Detecting intertrochanteric hip fractures with orthopedist-level accuracy using a deep convolutional neural network," by Urakawa and associates [5]. In this study, the authors compared the performance of convolutional neural network and five orthopedic surgeons in the radiographic diagnosis of intertrochanteric hip fractures of the proximal femur in 1773 patients. Collimated radiographs of the fractured proximal femur and hip (1773 cases) and non-fractured hip (1573 after exclusions) were used for the analysis. Using final clinical diagnoses based on all available imaging methods (including CT and MRI) as a standard of reference, the convolutional neural network and orthopedic surgeons had similar results with accuracies of $95.5 \%$ and $92.2 \%$, sensitivities of $93.9 \%$ and $88.3 \%$, specificities of $97.4 \%$ and $96.8 \%$, respectively; findings suggesting that artificial intelligence has the potential to screen radiographs, especially in the emergency room, where orthopedic surgeons are not readily available. To date, this manuscript has been cited 8 times.

Another highly cited scientific article, titled "Soft tissue aneurysmal bone cyst: six new cases with imaging details, molecular pathology, and review of the literature," by Song and colleagues [6], traces the history of this rare lesion. This manuscript highlights the clinical presentation of soft tissue aneurysmal bone cyst based on an analysis of all 36 published cases, the imaging features of the 12 cases with reported findings and the molecular analysis in the authors reported 6 cases. The results provide a succinct summary of this interesting and unusual lesion.
Another paper that caught the Editors' attention, as well as that of our readers was "Cementoplasty of pelvic bone metastases: systematic assessment of lesion filling and other factors that could affect the clinical outcomes," by Moser et al. [7]. With the increased use of percutaneous interventional techniques for the treatment of painful metastases as an alternative to surgery, this study highlighted techniques for the optimal filling of larger lesions with multiple needles; techniques which will likely be utilized with increasing frequency as the role of cementoplasty expands.

Although the Journal only published 5 perspectives in 2019 , two deserve special recognition. One that was especially interesting to readers as judged by its downloads and citations was "Revisiting tract seeding and compartmental anatomy for percutaneous image-guided musculoskeletal biopsies," by Leanne Seeger [8]. This short, easy-to-read perspective nicely summarizes the background and issues surrounding the controversy as to whether or not a biopsy tract should be considered contaminated tissue; whatever your belief, we suspect you will find this an interesting read! Another short perspective that was also highly downloaded and is well worth reading was titled "Incorporating musculoskeletal ultrasound into your radiology practice: patients, patience, and why your department cannot do without it," by Tara Morgan and Daria Motamedi [9]. The authors present how and why ultrasound has become an essential part of musculoskeletal imaging and why it should be incorporated into every practice.

No yearly review would be complete without some mention of the remarkable cases that we have the pleasure of seeing each month. One of the most frequently downloaded cases was "Hourglass-like constriction neuropathy of the suprascapular nerve detected by high-resolution magnetic resonance neurography: report of three patients," by Kim and colleagues [10]. This report describes three patients presenting with shoulder weakness in which a diagnosis of idiopathic neuralgic amyotrophy was suspected. The report illustrates the use of high-resolution neurography in these cases to confidently confirm a definitive diagnosis of hourglass-like constriction neuropathy as the underlying cause allowing conservative treatment.

Two "Test-Yourself" cases were also included in the most highly downloaded manuscripts. These both addressed unusual manifestations of clinical presentations we have all experienced. The first described a 29-year-old Caucasian female with a history of intravenous drug use' presenting with 1 week of worsening right hip and back pain, subjective fevers, chills, and lower back pain, titled "Worsening right hip pain," by Hussain et al. [11, 12], diagnosed with infected pseudoaneurysms. The second presents an isolated calcaneofibular ligament injury in a professional soccer player and was reported by Iqbal and associates [13, 14]; the manuscripts titled "Professional soccer player with an in-game ankle injury," focuses on this unusual injury which is nicely illustrated. 
The above articles were selected by the Editors from the list of the most commonly downloaded and cited articles selected by you, the Journal's readers. It has been a joy to have the opportunity to read them again during the preparation of this summary.

Before concluding, we would like to provide a brief update on the Journal inroads into the world of social media. The idea for posting Skeletal Radiology content on social media platforms was born in 2017 when a new ISS member reached out to the senior editors of the journal. The premise was simple: post content on Twitter to engage readers. This concept was presented at the Editorial Board during the 2017 International Skeletal Society Meeting in New York, NY, and Skeletal Radiology Social Media was born.

We began posting content on Twitter in January 2018. In our first year, we gained 460 followers. We decided to expand for January 2019, adding accounts on Instagram and Facebook. We post images from the papers published, as well as the DOI link to the manuscript. In 2019, we gained 264 followers on our Facebook page and 202 followers on our Instagram page. We saw the greatest gain on the Twitter account with 717 new followers in 2019 , for a total of 1177 . We currently have 265 followers on Facebook, 240 followers on Instagram, and 1386 followers on Twitter.

We have been fortunate to engage and interact with many readers on our social media platforms. We will continue to post high-quality content for all to enjoy. We hope you will join us!

\section{References}

1. Balius R, Pedret C, Iriarte I, Saiz R, Cerezal L. Sonographic landmarks in hamstring muscles. Skeletal Radiol. 2019;48(11):167583.

2. Kwee RM, Kwee TC. Calcified or ossified benign soft tissue lesions that may simulate malignancy. Skeletal Radiol. 2019;48(12): 1875-90.
3. Fields BKK, Skalski MR, Patel DB, White EA, Tomasian A, Gross JS, et al. Adhesive capsulitis: review of imaging findings, pathophysiology, clinical presentation, and treatment options. Skeletal Radiol. 2019;48(8):1171-84.

4. Tajmir SH, Lee H, Shailam R, Gale HI, Nguyen JC, Westra SJ, et al. Artificial intelligence-assisted interpretation of bone age radiographs improves accuracy and decreases variability. Skeletal Radiol. 2019;48(2):275-83.

5. Urakawa T, Tanaka Y, Goto S, Matsuzawa H, Watanabe K, Endo N. Detecting intertrochanteric hip fractures with orthopedist-level accuracy using a deep convolutional neural network. Skeletal Radiol. 2019;48(2):239-44.

6. Song W, Suurmeijer AJH, Bollen SM, Cleton-Jansen AM, Bovee J, Kroon HM. Soft tissue aneurysmal bone cyst: six new cases with imaging details, molecular pathology, and review of the literature. Skeletal Radiol. 2019;48(7):1059-67.

7. Moser TP, Onate M, Achour K, Freire V. Cementoplasty of pelvic bone metastases: systematic assessment of lesion filling and other factors that could affect the clinical outcomes. Skeletal Radiol. 2019;48(9):1345-55.

8. Seeger LL. Revisiting tract seeding and compartmental anatomy for percutaneous image-guided musculoskeletal biopsies. Skeletal Radiol. 2019;48(4):499-501.

9. Morgan TA, Motamedi D. Incorporating musculoskeletal ultrasound into your radiology practice: patients, patience, and why your department cannot do without it. Skeletal Radiol. 2019;48(2):1914.

10. Kim DH, Kim J, Sung DH. Hourglass-like constriction neuropathy of the suprascapular nerve detected by high-resolution magnetic resonance neurography: report of three patients. Skeletal Radiol. 2019;48(9):1451-6.

11. Hussain JA, Ali S, Montecalvo N, Panaro JN, Awan OA. Worsening right hip pain. Skeletal Radiol. 2019;48(4):621-3.

12. Hussain JA, Ali S, Montecalvo N, Panaro JN, Awan OA. Worsening right hip pain. Skeletal Radiol. 2019;48(4):649-50.

13. Iqbal A, McLoughlin E, Botchu R, James SL. Professional soccer player with an in-game ankle injury. Skeletal Radiol. 2019;48(10): 1621.

14. Iqbal A, McLoughlin E, Botchu R, James SL. Professional player with an in-game ankle injury. Skeletal Radiol. 2019;48(10):1657-8.

Publisher's note Springer Nature remains neutral with regard to jurisdictional claims in published maps and institutional affiliations. 